JOURNAL OF

APPLIED

CRYSTALLOGRAPHY

ISSN 1600-5767

\title{
Molecular Symmetry and Group Theory. Approaches in Spectroscopy and Chemical Reactions. By R. C. Maurya and J. M. Mir. De Gruyter, 2019. Pp. xiv+464. Paperback, price EUR 81.95, USD 94.99, GBP 74.50. ISBN 9783110634969.
}

\author{
Massimo Nespolo* \\ Université de Lorraine, CNRS, CRM2, Nancy, France. *Correspondence e-mail: massimo.nespolo@univ-lorraine.fr
}

Keywords: book reviews; molecular group theory; spectroscopy; chemical reactions.

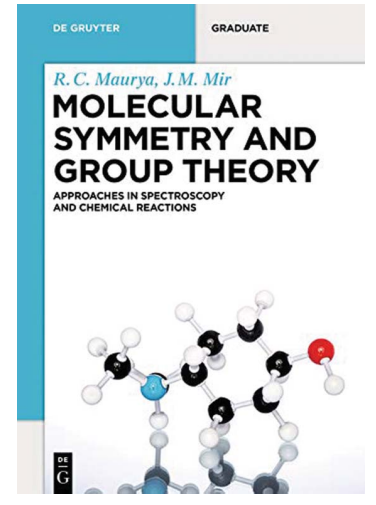

(C) 2020 International Union of Crystallography
Molecular Symmetry and Group Theory is co-authored by two Indian researchers with a foreword by the Vice-Chancellor of the Rani Durgavati University, Jabalapur. The title is quite ambitious and leads the reader to expect a reference text, which could well be the case, considering the almost 500 pages it contains. From the preface, however, some doubts arise. We read that 'It is only the last three decades or so that chemists are making use of this tool [group theory] to understand the fundamental concepts involved in bonding, structure of organic and inorganic compounds, spectral properties and occurrence of chemical reactions.' I dug out my copy of Cotton's Chemical Applications of Group Theory to check the publication date: the first edition came out in 1963, that is 56 years before the publication of the textbook reviewed here. Note that Cotton's classical textbook is cited (although in its 3rd edition) as reference No. 1 in the bibliography: the above statement is therefore utterly puzzling. This suggested to me to approach my critical review by having Cotton's classical textbook at hand, to see what else is covered here and what more is present for the topics that are in common. The result is a dismissal with prejudice: this most recent textbook cannot measure up to its predecessor. One of the main defects of the new book is the extensive use of concepts and terms that are not defined, so that the beginner would need another book to understand what the authors write about. As a matter of fact, the book is mainly composed of a set of examples where unexplained 'how-to-do' rules are applied again and again.

The descriptive example-driven approach could be appreciated if coming as a complement to a rigorous introduction and definition. These are instead systematically missing and the reader is expected to guess the meaning of the method presented, or may just learn how the machinery works as an article of faith. Although the target public starts at the master's level, it is unclear what background is assumed. Considering the huge number of terms and concepts that are used without being introduced, defined and explained, one feels sympathy with students who have to cope with content that has more holes than a piece of Emmental cheese.

The first chapter (27 pages) introduces the very basic notions of molecular symmetry, through a number of examples in which the symmetry operations and symmetry elements of different molecules are discussed. We find here the widespread confusion between geometric element and symmetry element. Note that the concept of group is absent from this chapter, but extensively used from the following chapter. Whether it has simply been forgotten or the authors do not consider it necessary to explain what a group is remains an unsolved mystery. Molecules with the shape of trigonal bipyramids or trigonal planar molecules are among the many examples provided, but nowhere is the term 'trigonal' introduced or defined.

The second chapter (113 pages, of which most are worked-out examples) bears the title Application of group theory to electronic spectroscopy. Fundamental concepts are extensively used which have not been introduced, defined or explained: representations, reducibility and irreducibility, direct product of representations, character of an operation (one has to wait until page 213 in the following chapter to eventually get a definition), conjugacy classes, Mulliken symbols...: all the traditional weaponry of group theory shows up as if the reader woke up in the middle of a TV series of which he or she had missed half of the episodes! But that is not all: without any definition or explanation, 
concepts pop up, like the transition moment integral wavefunction, dipole moment operator, Franck-Condon principle, orthogonal set of functions, degeneration, crossover point .... Is the reader expected to know everything in advance? This seems unreasonable, considering the target public. One is left with the impression that the book is not meant to explain anything, just to show 'how it works' (e.g. 'some rules are to be known': page 61). Accordingly, the 'reduction formula' to get the number of irreducible representations in terms of which a reducible representation can be decomposed shows up at page 42 like a deus ex machina: Schur orthogonality relations are not even mentioned. The symbol $\Delta$ occurs at page 49 but nowhere is it explained that it represents the crystal field stabilization energy: readers must know it! Following the same trend, Russell-Saunders coupling and $j-j$ coupling are used on the next page without any definition of the angular momenta $l$, $s$ or $j$. Again, is the exchange energy mentioned at page 57 known to all readers of a book of molecular symmetry which addresses master's students? Crystal field theory (CFT at page 60: if you do not know it already you won't guess) and ligand field theory (page 61) are among the concepts that the authors do not consider necessary to explain; when readers come to the distinction between strong field and weak field (page 90) they must feel completely lost (unless they know already ... but then why read this particular book?). After a long series of examples, we arrive at a section about Tanabe-Sugamo diagrams, where Racah parameters are used without ever being introduced, defined or explained. The chapter goes on for several more pages with guided exercises, which are absolutely impossible to follow if the reader does not already know what is behind them, and practically useless if he or she knows already.

Chapter 3 (234 pages) has the title Molecular symmetry and group theory to vibrational spectroscopy (is 'applied' missing?). After the introduction of local references on each atom of a simple molecule, we find some ideas about reducible and irreducible representations, although the treatment is insufficient because the rigorous approach available in many textbook would be 'very cumbersome and time-consuming' (page 152). One wonders why, although very limited and insufficient, this presentation has been postponed so late in the book, while the concepts lightly introduced here are used already in the previous chapter. The whole chapter is essentially a long list of worked-out examples of construction of reducible representations, reduction to irreps, and determination of IR and Raman active modes: nothing more than what is found in Cotton's classical textbook, with the difference that here one simply gets a recipe, without any background to understand the physical reasons behind it. Note that the $\mathrm{POCl}_{3}$ molecule is wrongly addressed as 'tetrahedral', whereas its point group is $C_{3 v}(3 m)$, which shows that the symmetry is trigonal pyramidal. An intermission within the tedious list of examples is taken with some pages about overtones, combination bands, hot bands, always in an apodictic and confusing way. As an example, let us consider the following sentence about Fermi resonance (page 324): 'Although, the interaction is in between different kinds of bands but due to approximatively same energy and same symmetry we may call them accidentally degenerate'. No comment...

The fourth and last chapter (64 pages), under the title Chemical reaction: orbital symmetry rules, covers some content normally present in textbooks of organic chemistry. Symmetry considerations are used essentially to predict the type of atomic orbital overlap to form bonding and anti-bonding molecular orbitals; no real group theory is used in this chapter, with one exception to predict 'stable shape of molecules'. One may reasonably wonder why it had to be inserted in this book.

Each chapter ends with exercises and 'type questions', but the answers are not given. Three appendices close the book, presenting character tables of 'chemically important symmetry groups', a correlation table (of irreps) for $O_{h}$ and $T_{d}$ groups, and some trigonometric relations.

A number of typos show that the proofreading stage was absent or far from rigorous: "number of times... smallest rotation' instead of 'smallest number of times... rotation' (page 4), 'reflection' instead of 'inversion' (page 16), $\mathrm{N}_{2} \mathrm{~F}_{2}$ instead of $\mathrm{N}_{2} \mathrm{~F}_{4}$ (page 18), $\sigma h$ instead of $\sigma_{h}$ (page 19, twice), 'lower filled... higher vacant' instead of the opposite, as should be evident from the acronyms HOMO and LUMO (page 29), 'Mo' instead of 'MO' (page 35), 'fee ion' instead of 'free ion' (page 69), 'planer' instead of 'planar' (page 229), number of figure missing (page 331), 'Taman' instead of 'Raman' (page 354), 'LUMP' instead of 'LUMO' (page 412), and some plural/singular mistakes here and there.

Going through a book like this is tantamount to crossing the Styx. At the end of such a path one question rises into this reader's mind. How is it possible that a serious scientific publisher sends to the press a manuscript which should not pass even the first step of peer review? Unfortunately, it appears that textbooks and monographs are too often accepted on submission of a simple plan and table of contents, without sufficient peer review. In my opinion, there is an urgent need to change the publication model, otherwise manuscripts like this, which jeopardize the reputation of the publishing house, will continue to go through. Marketing considerations must never take priority over science. 